\title{
Purificación de lecitina de soja. 1-Selección de equipamiento y determinación de las condiciones operativas. 2-Obtención de diagrama de equilibrio
}

\author{
Por B. Maroto*, R. Madoery y C. Camusso \\ Laboratorio de Química Orgánica. Dpto. de Biología Aplicada. \\ Facultad de Ciencias Agropecuarias. Universidad Nacional de \\ Córdoba. C.C. 509-5000 Córdoba. REPUBLICA ARGENTINA.
}

\section{RESUMEN}

Purificación de lecitina de soja. 1-Selección de equipamiento y determinación de las condiciones operativas. 2-Obtención de diagrama de equilibrio.

En el presente trabajo se describe la selección de las condiciones operativas óptimas para la purificación de lecitina de soja, utilizando acetona y operando en discontinuo.

El equipo se diseñó teniendo en cuenta que se producia una gran variación de la viscosidad que dificultaba la extracción, por lo que se debió prestar especial atención al diseño del sistema impulsor y del sistema agitador.

Operando bajo estas condiciones se obtuvieron los valores de equilibrio para la mezcla fosfolipidos/aceite/acetona.

Los resultados evidencian interacción entre los fosfolípidos y el aceite, bien porque habría absorción preferente de soluto o bien porque el soluto es soluble en el sólido y no se distribuye por igual entre las fases líquidas y sólidas en el equilibrio.

PALABRAS-CLAVE: Condiciones de trabajo - Diagrama de equilibrio - Equipamiento - Lecitina de soja - Purificación.

\section{SUMMARY}

Purification of soybean lecithin. 1-Equipment and operating conditions selection. 2-Equilibrium diagrams.

In this work we describe the choice of the best operating conditions to produce pure powder soybean lecithin, extracting with acetone, in a bench scale.

The equipment was designed in order to obtain adequate impeller and turbine systems response because there is significant viscosity variations between batches, and the extraction process was difficult.

By operating in this conditions, we have obtained the equilibrium values for the phospholipids/oil/acetone mixture.

The results showed that there are a phospholipids/oil interaction and this might be by solute absorption or because the solute is soluble in the solid and it is no reparting by equal between the equilibrium liquid and solid phases.

KEY-WORDS: Equilibrium diagram - Equipment - Operating conditions - Purification-Soybean lecithin.

\section{INTRODUCCION}

La investigación en tecnología de alimentos es uno de los puntos de mayor impacto sobre la actividad socioeconómica del país. A ello contribuye, no sólo el hecho de poseer la República Argentina una importante producción primaria que requiere su industrialización, con el consiguiente aporte de tecnología, sino que también el avance mundial en tecnología de alimentos se encuentra en un estado incipiente.

La producción de aceite de soja en la Provincia de Córdoba alcanza las 70.000 Ton/año: esto implica una producción de 4.000 Ton/año de gomas crudas húmedas (lecitinas impuras) que deben ser procesadas o tratadas como efluentes contaminantes. Parte de estas gomas son deshidratadas y exportadas como tales, el resto es mezclado con harinas para su utilización como alimento equilibrado (1).

No existen en el país industrias que procesen las gomas crudas secas con el fin de obtener lecitina pura o refinada. La aceptación del producto LECITINA DE SOJA PURA EN POLVO, no sólo estará dada por su funcionalidad y calidad sino también por los costos que implica su purificación; de allí habría dos alternativas: una, el uso de las gomas crudas secas y otra, el empleo de las lecitinas purificadas (2).

La bibliografía registra la preparación de fosfátidos en polvo a partir de aceite de soja por un procedimiento que parte del desgomado, el centrifugado, el secado y la precipitación con acetona de los fosfátidos, resultando un polvo amarillento (3).

Se desarrolló un procedimiento de purificación de lecitina de soja, logrando purificar a nivel laboratorio las gomas crudas secas por sucesivas extracciones con acetona, obteniéndose lecitina pura en polvo, la cual fue caracterizada químicamente (4) (5) (6).

En el presente trabajo se describe el diseño y optimización en operación de un equipo de agitaciónextracción. El problema consistió en la extracción del aceite de soja utilizando acetona como solvente, pero teniendo en cuenta que el sistema en una primera etapa 
se comportaba como monofásico perfecto, y a medida que se avanzaba en el proceso de purificación la viscosidad aumentaba dificultando la extracción. La estructura liquido-cristalina de los fosfolípidos, que les permite adoptar diferentes disposiciones según sea la concentración lipídica, sería la responsable de este comportamiento (7). Para cumplir con la condición de mantener el mismo nivel de agitación en todas y cada una de las etapas, a pesar de ese brusco incremento de viscosidad que se experimenta en una etapa intermedia de la extracción, el tratamiento del diseño y cambio de escala para el equipo en discontinuo (micro planta piloto), debió contemplar el diseño del sistema impulsor y del sistema agitador. Cumplida esta etapa de diseño y optimización del equipo de agitación-extracción se obtuvieron los valores de equilibrio en operación para el sistema fosfolípidos/ aceite de soja/acetona.

\section{DISEÑO DEL EQUIPO} (8).

Se siguieron las etapas de diseño especificadas en

\section{MATERIALES Y METODOS}

El equipo de agitación-extracción se construyó enteramente en acero inoxidable. Esta constituído por un tanque de agitación cilíndrico con fondo torisférico y concéntrico con él, y en posición vertical, posee un eje accionado por un motor-reductor a polea en cuyo extremo se encuentra la turbina de agitación, con cuatro paletas inclinadas a $45^{\circ} \mathrm{C}$.

Todas las operaciones de carga y descarga se realizaron por el extremo abierto del tanque y siempre bajo campana extractora de gases.

El porcentaje de agua en el solvente de extracción se determinó por conductimetría. Se siguió la evolución de la extracción cuantificando insolubles en acetona (IA) (9).
Se utilizaron gomas crudas secas comerciales con la siguiente composición promedio:

Fosfolípidos totales (insolubles en Acetona) $\quad 60-65 \%$

Aceites $30-35 \%$

Humedad

Impurezas $3 \%$

Por experiencias previas (2) (3) (4), se pudo determinar que el solvente extractor más conveniente para este proceso era acetona, en sus grados comercial y bidestilado, así como la relación sólido/líquido y el número de extracciones, para un sistema a escala laboratorio de operación manual.

El producto obtenido, LECITINA PURA EN POLVO, rindió $97 \%$ de fosfolípidos totales y fue desolventizado en estufa de vacío a $50^{\circ} \mathrm{C}$.

Se programaron dos series de ensayos:

\section{a) Operaciones tipo A:}

Para cada operación de extracción se realizaron cinco etapas consecutivas, empleando en todos los casos una relación sólido/líquido $1: 1$ (1250 g de lecitina: 1250 $\mathrm{ml}$ de acetona) y con tiempos de agitación variables, de 15 y 30 minutos por cada etapa.

\section{b) Operación tipo $B$ :}

Se realizaron cinco etapas de extracción con tiempos de 15 y 30 minutos por etapa, pero la diferencia con las operaciones tipo $\mathrm{A}$ radica en la relación sólido/líquido, que no fue constante a lo largo de todo el proceso.

La relación sólido/líquido elegida fue 1:0,75 para la primera etapa de extracción y luego 1:0,5 para las etapas siguientes. Se determinó que la relación 1:0,75 fue la mínima posible para la primera etapa, que permitió una separación adecuada de las fases.

Los resultados obtenidos en las operaciones tipo A se resumen en la Tabla I, para las etapas de 15 minutos y en la Tabla II para las etapas de 30 minutos.

Operaciones tipo "A». Extracciones de 15 minutos cada etapa.

\begin{tabular}{|c|c|c|c|}
\hline $\begin{array}{c}\text { Número de } \\
\text { extracción. }\end{array}$ & $\begin{array}{c}\text { Tiempo total } \\
\text { de extracción }\end{array}$ & IA & $\begin{array}{c}\text { Relación } \\
\text { sólido/1iquid }\end{array}$ \\
\hline 0 & 0 & 60 & - \\
\hline 1 & 15 & 71 & $1: 1$ \\
\hline 2 & 30 & 82 & $1: 1$ \\
\hline 3 & 45 & 89 & $1: 1$ \\
\hline 4 & 60 & 93 & $1: 1$ \\
\hline 5 & 75 & 95 & $1: 1$ \\
\hline 6 & 90 & 97 & $1: 1$ \\
\hline
\end{tabular}


Tabla II

Operaciones tipo «An. Extracciones de 30 minutos cada etapa.

\begin{tabular}{|c|c|c|c|}
\hline $\begin{array}{c}\text { Número de } \\
\text { extracción. }\end{array}$ & $\begin{array}{c}\text { Tiempo total } \\
\text { de extracción }\end{array}$ & IA & $\begin{array}{c}\text { Relación } \\
\text { sólido/liquid }\end{array}$ \\
\hline 0 & 0 & 59 & - \\
\hline 1 & 30 & 70 & $1: 1$ \\
\hline 2 & 60 & 80 & $1: 1$ \\
\hline 3 & 90 & 88 & $1: 1$ \\
\hline 4 & 120 & 92 & $1: 1$ \\
\hline 5 & 150 & 95 & $1: 1$ \\
\hline 6 & 180 & 97 & \\
\hline
\end{tabular}

Los resultados obtenidos en las operaciones tipo B se resumen en la Tabla III para las etapas de 15 minutos y en la tabla IV para las etapas de 30 minutos.

\section{INTERPRETACION DE LOS RESULTADOS}

La representación gráfica de las Tablas I y II, como de las Tablas III y IV, conducen a curvas de tipo exponencial, que tienden a un valor asintótico próximo al 97\% en IA (fosfolípidos totales), cuando se representa IA frente al tiempo de agitación. Estos valores experimentales se ajustan perfectamente a una ecuación como la siguiente, en que se ha rectificado la expresión de la curva:

$$
\ln (100-\mid A . t)=m t+\ln \left(\mid A . t_{0}\right)
$$

donde "IA" corresponde al porcentaje de fosfolípidos obtenidos hasta esa etapa y « $\mathrm{m}$ » al coeficiente angular de la recta.

Tabla III

Operaciones tipo «B». Extracciones de 15 minutos cada etapa.

\begin{tabular}{|c|c|c|c|}
\hline $\begin{array}{l}\text { Número de } \\
\text { extracción. }\end{array}$ & $\begin{array}{l}\text { Tiempo total } \\
\text { de extracción }\end{array}$ & IA & $\begin{array}{l}\text { Relación } \\
\text { sólido/liquid }\end{array}$ \\
\hline 0 & 0 & 60 & - \\
\hline 1 & 15 & 70 & $1: 0,75$ \\
\hline 2 & 30 & 78 & $1: 0,5$ \\
\hline 3 & 45 & 85 & $1: 0,5$ \\
\hline 4 & 60 & 90 & $1: 0,5$ \\
\hline 5 & 75 & 94 & $1: 0,5$ \\
\hline 6 & 90 & 97 & $1: 0,5$ \\
\hline
\end{tabular}

Tabla IV

Operaciones tipo “B». Extracciones de 30 minutos cada etapa.

\begin{tabular}{|c|c|c|c|}
\hline $\begin{array}{l}\text { Número de } \\
\text { extracción. }\end{array}$ & $\begin{array}{l}\text { Tiempo total } \\
\text { de extracción }\end{array}$ & IA & $\begin{array}{l}\text { Relación } \\
\text { solido/liquid }\end{array}$ \\
\hline 0 & 0 & 60 & $1: 0,75$ \\
\hline 1 & 30 & 71 & $1: 0,5$ \\
\hline 2 & 60 & 80 & $1: 0,5$ \\
\hline 3 & 90 & 86 & $1: 0,5$ \\
\hline 4 & 120 & 92 & $1: 0,5$ \\
\hline 5 & 150 & 95 & $1: 0,5$ \\
\hline 6 & 180 & 97 & 1.07 \\
\hline
\end{tabular}


Analizando los resultados anteriormente expuestos, se concluye que el proceso de extracción no depende del mayor o menor tiempo por etapa y sí, del número de ellas.

De la ecuación anterior, se deduce el tiempo teórico necesario para obtener el $100 \%$ de lecitina de soja, en cada serie de experiencias, resultando:

- para extracciones de 15 minutos cada etapa: tiempo total de extracción 136 minutos.

- para extracciones de 30 minutos cada etapa: tiempo total de extracción 262 minutos.

Se infiere entonces, que el proceso de extracción discontinuo óptimo para el sistema extractor sería el indicado en la figura $A$, ya que en él se ven optimizados tanto los tiempos de extracción, como la relación sólido/ líquido (Tablas I y III).

\section{MEDICION DE VALORES DE EQUILIBRIO}

Teniendo en cuenta el sistema óptimo para la extracción del aceite de soja con acetona (figura A) se analizó el contenido de acetona, aceite de soja y fosfolípidos en los extractos y refinados provenientes de cada etapa de extracción, así como el producto obtenido, para luego resumirlos en la figura $B$, en la que se representa por medio de un diagrama rectangular, en ordenadas: $\mathrm{kg}$ de inerte / $\mathrm{kg}$ de disolución, $(\mathrm{N}=\mathrm{B} /(\mathrm{A}+\mathrm{C})$; $y$ en abscisas: $\mathrm{kg}$ de soluto / $\mathrm{kg}$ de disolución, $(X, Y=C /(A+C))(11)$.

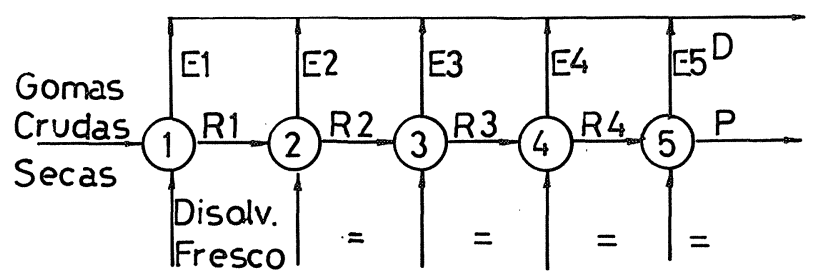

Figura A

Esquema del proceso de extracción discontinuo.

- 1,2,3,4,5 representan una etapa de extracción de 15 minutos.

- R1,R2,R3,R4 los refinados provenientes de las etapas correspondientes.

- E1,E2,E3,E4,E5 los extractos que provienen de las correspondientes etapas de extracción.

- P es la lecitina de soja que se envía a la etapa de secado

- D es la suma de los extractos que se envía a la etapa de recuperación.

Considerándose a los fosfolípidos como el inerte B que no puede extraerse con acetona, $C$ el soluto a extraer, aceite de soja, y A el solvente extractor, acetona.

La disolución retenida por unidad de sólido inerte viene representada por la curva KG. La línea K'G' representa la composición de las soluciones provenientes de las distintas etapas. Los segmentos tales como AA', BB', etc., representan las líneas de unión que permiten construir la figura $C$, es decir $Y$ frente $X(C /(A+C)$ en los lodos frente a $C /(A+C)$ en la solución).
Figura B

Equilibrio práctico para el sistema aceite de soja/fosfolípidos/acetona.

$$
\mathrm{N}=\mathrm{B} /(\mathrm{A}+\mathrm{C})=\mathrm{Kg} \text { de inerte } / \mathrm{Kg} \text { de disolución. }
$$

$X, Y=C /(A+C)=K g$ de soluto $/ K g$ de disolución.

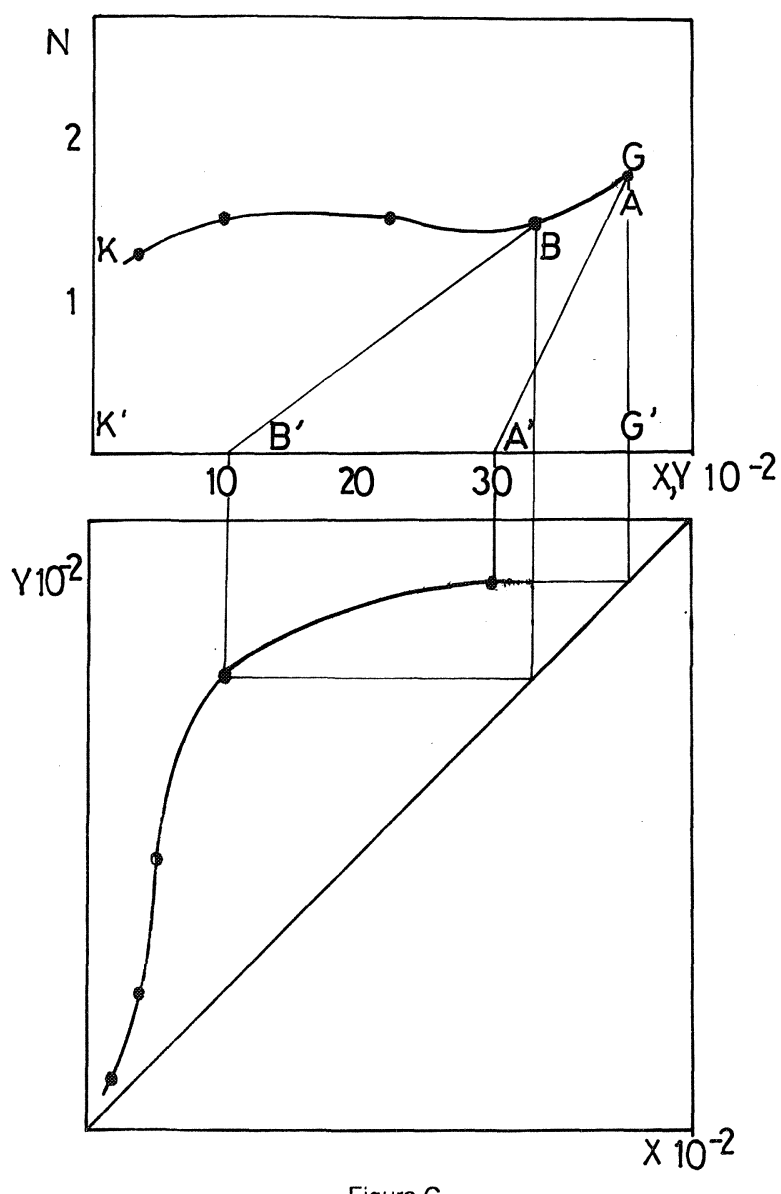

Figura C

Composiciones de equilibrio para el sistema aceite de soja/fosfolipidos/ acetona.

$Y=C /(A+C)=K g$ de soluto $/ \mathrm{Kg}$ de disolución, en los lodos.
$X=C /(A+C)=K g$ de soluto $/ \mathrm{Kg}$ de disolución, en la solución

\section{CONSIDERACIONES FINALES}

A partir de los ensayos realizados, se concluye que el factor determinante en el proceso de extracción de aceite de soja de las gomas crudas secas, no es la solubilidad del aceite en la acetona. Esta afirmación surge de observar que si el aceite se encontrara simplemente disuelto en la lecitina, un simple contacto entre las gomas crudas secas y el disolvente, haría sencilla su extracción. La experiencia nos demuestra, que se hace necesaria una agitación efectiva para extraer el aceite. Algo similar ocurre en la extracción de orujos procedentes del sistema ALFIN, donse se ha observado que al ponerse en contacto el orujo con el disolvente, el "aceite suelto" se disuelve en forma casi instantánea, pero para extraer el 
"aceite ocluído" se hace necesaria una agitación efectiva, hasta que a tiempo infinito se lograría un equilibrio cuando la miscela exterior tuviese la misma concentración que la interior (10).

Se deduce entonces, que una porción de aceite efectivamente se encuentra disuelta en la lecitina, pero otra fracción bastante importante se halla relacionada con ella interaccionando química o fisicoquímicamente, lo que impide que el simple contacto con el solvente, haga posible su separación de los fosfolípidos que integran las gomas crudas secas.

Del análisis de las figuras $B$ y $C$ surge que (12):

- el aceite de soja es infinitamente soluble en la acetona, de forma tal que $\mathrm{X}$ e $\mathrm{Y}$ pueden tomar valores que van de 0 a 1.

- la curva KG representa el sólido separado en las condiciones reales previstas para la práctica, como ya se dijo, y nos indica que los sólidos no poseen el mismo grado de sedimentación o drenaje en todas las concentraciones de soluto.

- K' G' nos indica que la solución no contiene sustancia $B$, ni disuelta ni suspendida.

- las líneas de unión no son verticales, esto ocurre ya sea, porque hay absorción preferente del soluto 0 , porque el soluto es soluble en el sólido $B$ y no se distribuye por igual entre las fases líquida y sólida en el equilibrio.

\section{AGRADECIMIENTOS}

Para la realización de este proyecto se ha recibido el apoyo económico de CONICOR (Res. 1033/88, 1244/ 89), SECYT (Res. 323/87, 411/88) y CAPFTA (1936/90).

\section{BIBLIOGRAFIA}

1. Consejo Federal de Inversiones. Fundación Banco de la Provincia de Córdoba. Desarrollo Agroindustrial de la Provincia de Córdoba: 6-1 y 6-2: Relevamiento de la producción agroindustrial. 1984.

2. Madoery, R., Abril, A., Cabrol, R., Camusso, C. y Maroto. B. - Purificación de lecitina de soja".- III Congreso Latinoamericano de Cs. Farmacéuticas. Montevideo, 1986.

3. Abril, A., Camusso, C. y Madoery, R. -„Procedimiento para la obtención de lecitina pura en polvo a partir de gomas crudas secas".- Dirección Nacional de la Propiedad Industrial. Acta 309509. 7/12/87.

4. Madoery. R., Abril, A., Cabrol, R., Camusso, C. y Maroto, B - Purificación de lecitina de soja 1- Obtención de lecitina pura en polvo. 2 Obtención de fosfatidilcolina pura».- La Alimentación Latinoamericana 163 (1987) 60-63.

5. Van Nieuwenhuyzen, W. -"Lecithin production and properties".- J. Am. Oil Chemists' Soc. 53 (1976) 425-427.

6. Kristappa, G., Azeemodding, G. and Thirumala Rao, S.D. - Powder phosphatides from indian soybean oil».- Paintindia 26 (8) (1976) 21-22.

7. Chapman, D. -"Lipidos".- Editorial Alhambra. 1972.

8. Gates, L.E., Henley, T.L. and Fenic, J.G. - How to select the optimum turbine agitator".- Chem. Eng. Dec. 8 (1975) 110-114.

Dickey, D.S. and Fenic, J.G. - -Dimensional analysis for fluid agitation systems".- Chem, Eng. Jan. 5 (1976) 139-145.

Dickey, D.S. and Hicks, R.W. --Fundamentals of agitation".- Chem. Eng. Feb. 2 (1976) 93-100.

Hicks, R.W., Morton, J.R. and Fenic, J.G. - "How to design agitator for desired process response".- Chem. Eng. April 26 (1976) 102-110.

9. "Quality assessment of lecithin".- Lucas Meyer -Hamburg.

10. Flores Luque, V., López Romasanta, F.C., Gómez Herrera, C. y Pereda Marín, J. - La agitación en la extracción con disolvente de orujos procedentes de sistemas ALFIN. 1-Generalidades de la cinética del proceso".- Grasas y Aceites 19 (1968) 247-251.

11. Ocon-Tojo. -„Problemas de Ingeniería Química. Operaciones básicas."- Tomo 2.- Editorial Aguilar, 1970.

12. Trebial, R. -»Mass transfer operations».- Ed. Mc. Graw Hill, NY, 1968. 